\title{
Conocimiento y percepción acerca del cambio climático en comunidades costeras del municipio de Guasave, Sinaloa, México
}

\author{
Knowledge and perception about climate change in coastal communities \\ in the municipality of Guasave, Sinaloa, Mexico
}

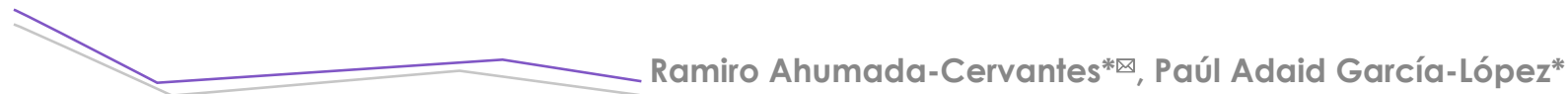

Ahumada-Cervantes, R., \& García-López, P. A. (2018). Conocimiento y percepción acerca del cambio climático en comunidades costeras del municipio de Guasave, Sinaloa, México. Investigación y Ciencia de la Universidad Autónoma de Aguascalientes, 26(75), 38-45.

\section{RESUMEN}

El cambio climático es un problema de actualidad que requiere acción urgente, se presenta un estudio con el objetivo de analizar el nivel de conocimiento y percepción acerca del fenómeno estudiado en tres comunidades costeras del municipio de Guasave, Sinaloa, México. La técnica utilizada para obtener la información fue la aplicación de encuestas, en total 193 cuestionarios que se enfocaron en temas como la conceptualización del cambio climático, su impacto en los medios de vida, entre otros. Los resultados revelan que un elevado porcentaje de personas percibe un cambio en el clima, principalmente en la temperatura y que aproximadamente en un cuarto de la población encuestada se percibe un nivel de conocimiento aceptable del tema. Los ciudadanos se muestran receptivos para recibir información relacionada con el mismo y para implementar estrategias de mitigación y adaptación.

Palabras clave: cambio climático; comunidades costeras; conocimiento; contaminación; pesca; percepción.

Keywords: climate change; coastal communities; fishing; knowledge; perception; pollution.

Recibido: 19 de octubre de 2017, aceptado: 11 de junio de 2018

* Departamento Académico de Ingeniería y Tecnología, Universidad Autónoma de Occidente Unidad Regional Guasave. Avenida Universidad s/n, Fraccionamiento Villa Universidad, C. P. 81048, Guasave, Sinaloa, México. Correo electrónico: ramiroac2002@yahoo.com.mx; pauladaid@hotmail.com
ABSTRACT

As has been stated previously that climate change is a topical problem that requires urgent action, a study is presented with the objective of analyzing the level of knowledge and perception about climate change in three coastal communities in the municipality of Guasave, Sinaloa, Mexico. The technique used was the conducting surveys, a total of 193 questionnaires were applied. The questions focused on topics such as the conceptualization of climate change, the impact of climate change on livelihoods, among others. The results show that a high percentage of people perceive a change in climate, mainly in temperature and that approximately, in a quarter of the population surveyed a level of acceptable knowledge about climate change is perceived. Citizens are receptive to receive training and to implement mitigation and adaptation strategies.

\section{INTRODUCCIÓN}

El cambio climático (CC) es una transformación del clima por influencia de la actividad humana que altera la composición de la atmósfera global y que se suma a la variabilidad natural del clima observada durante periodos de tiempo comparables (SEMARNAT, 2012).

El calentamiento global es innegable y se observa tanto en la atmósfera, como en el océano. Está provocando que los volúmenes de nieve y hielo disminuyan y que el nivel del mar se eleve (Panel Intergubernamental de Cambio Climático [IPCC, por sus siglas en inglés], 2014). Estos cambios biofísicos, 
en correspondencia con los socioeconómicos en distintas escalas y velocidades, interactúan para producir diferentes resultados para las comunidades en las distintas regiones (Bennett, Blythe, Tyler, \& Ban, 2016; Tuler, Agyeman, Da Silva, LoRusso, \& Kay, 2008).

Es posible que las comunidades costeras sean afectadas por el CC en aspectos como aumento del nivel del mar, aumento de la frecuencia e intensidad de los eventos extremos, así como cambios en la disponibilidad de los recursos pesqueros (Bennett et al., 2016; Colburn et al., 2016; Nanlohy, Bambangb, Ambariyanto, \& Hutabarat, 2015).

En este sentido, el CC pone de manifiesto la vulnerabilidad de las comunidades expuestas a sus impactos; sin embargo, no se debe únicamente a las presiones naturales, sino que es agravada por los procesos sociales, económicos y políticos reinantes en cada región (Soares \& García, 2014). Para analizar la vulnerabilidad de la población ante el suceso en estudio es necesario comprender su interacción con los medios de sustento, las características sociodemográficas de la población, así como la percepción que la misma tiene con respecto al problema (Haq \& Ahmed, 2017; Sandoval, Soares, \& Munguía, 2015).

El IPCC (2014) señala que existen diversos métodos de estudio para la generación de información que conduzca a la toma de decisiones encaminadas a limitar el CC y sus efectos, entre ellos, la percepción y respuesta de la población ante el riesgo y la incertidumbre. La percepción juega un papel fundamental para que la sociedad demande estrategias enfocadas en la mitigación y adaptación, a la vez que se involucra en el proceso (Arbuckle, Morton, \& Hobbs, 2013; Meira-Cartea, 2008). La investigación en CC requiere del estudio de la percepción que tiene la población involucrada acerca del fenómeno, ya que la implementación de cualquier estrategia o política exige conocer su nivel de conocimiento y comprensión del problema, así como su disposición para favorecer la implementación de dicha política (Carlton \& Jacobson, 2013; Hagen, Middel, \& Pijawka, 2016; Retamal, Rojas, \& Parra, 2011).

El enfoque de la percepción acerca del CC en comunidades costeras ha sido abordado en diversos estudios (Nanlohy et al., 2015; Olmos Martínez, González Ávila, \& Contreras Loera,
2013; Shameem, Momtaz, \& Kiem, 2015; Soares \& Gutiérrez, 2011; Soares \& Murillo-Licea, 2013) y ha evidenciado el poco conocimiento de la población sobre los aspectos relacionados con el fenómeno. Esta falta de conocimiento es más acentuada en lo concerniente a comunidades rurales.

Al ser consideradas de las más vulnerables ante los embates del CC, es una necesidad imperante abordar su impacto en comunidades costeras, en especial su percepción acerca del problema. Lamentablemente un gran porcentaje de la población residente no comprende lo que es el CC, la manera como podría ser impactada por este fenómeno, así como las estrategias que podrían implementar para minimizar sus impactos.

A partir de lograr que las personas sean conscientes de la existencia, el impacto y las causas del CC, será posible integrarlo en la planificación e implementación de políticas, proyectos y actividades diarias, participando en el proceso de mitigación y adaptación. Por tal razón, el objetivo del presente estudio fue analizar el nivel de conocimiento y percepción acerca del CC en tres comunidades costeras del municipio de Guasave, Sinaloa, México. Las comunidades involucradas son de tipo rural y su principal actividad productiva es la pesca.

\section{MATERIALES Y MÉTODOS}

\section{Descripción del área de estudio}

La región de estudio comprende las localidades El Coloradito, El Caracol y El Huitussi (figura 1). Corresponde a comunidades rurales localizadas en la zona costera del municipio de Guasave, Sinaloa. Dicho municipio está localizado en el norte del estado, entre los paralelos $25^{\circ} 11^{\prime}$ y $25^{\circ} 50^{\prime}$ norte y los meridianos $108^{\circ} 10^{\prime}$ y $109^{\circ} 02^{\prime}$ oeste (INEGI, 2009).

El Huitussi tiene la mayor población, cuenta con 2,286 hab; El Caracol con 999 y El Coloradito con 307. La población económicamente activa asciende a $39.5 \%$ en El Coloradito, a $38.2 \%$ en El Caracol y a $38 \%$ en El Huitussi; desempeñan principalmente actividades relacionadas con pesca, agricultura y ganadería (respectivamente). Las tres comunidades cuentan con una tasa de alfabetización arriba de $93 \%$ y una asistencia escolar por población de 6 a 14 años arriba de $95 \%$. Existen algunos rezagos en relación con la disponibilidad de servicios en 


\section{INVESTIGAGIÓก Y CIECEIA DE LA UחIVERSIDAD AUTÓกOMA DE RGUASCALIERTES}

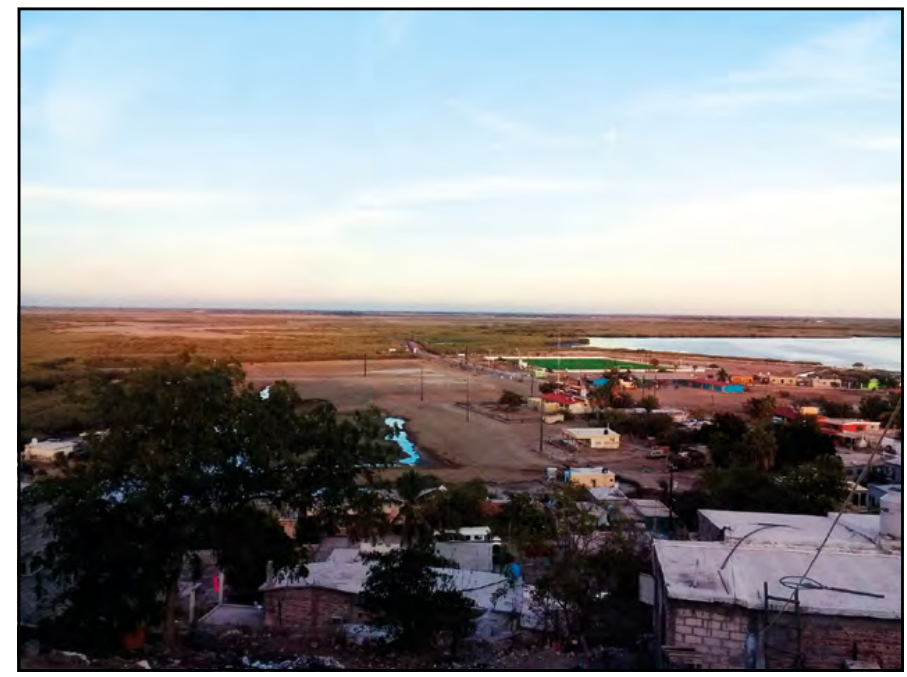

Figura 1. Comunidad de El Huitussi.

Imagen de Ramiro Ahumada Cervantes.

las viviendas, principalmente en agua potable y drenaje, El Coloradito es la de mayor rezago (INEGI, 2010).

El tipo de clima es muy seco, cálido y cálido -BW(hı)- con lluvias en verano y un porcentaje de lluvias en invierno entre $5 \%$ y $10.2 \%$ del total anual, con temperatura media anual mayor a $22{ }^{\circ} \mathrm{C}$. El tipo de suelo dominante es Solonchak, inundable en algunas épocas del año y la vegetación es de matorral, principalmente manglar (INEGI, 2009).

\section{Aplicación de encuestas}

La información se obtuvo mediante la aplicación de encuestas personales (figura 2). Se utilizó un cuestionario combinado, el cual consistió en cuatro preguntas cerradas y seis abiertas.

Para explorar la percepción de los pobladores relativa a cambios en el clima en los últimos años, se utilizaron dos preguntas: 1. ¿ेPercibe algún cambio en el clima en los últimos años? 2. ¿En qué aspectos del clima percibe estos cambios? Únicamente a quienes respondieron positivamente la primera pregunta se les cuestionó la segunda.

En cuanto al nivel de conocimiento que los pobladores tienen acerca del CC, se utilizaron seis preguntas. La primera se enfoca en la sensibilización o información que los encuestados han recibido en materia de CC.

La segunda se refiere a la configuración de los habitantes con respecto al tema estudiado, lo

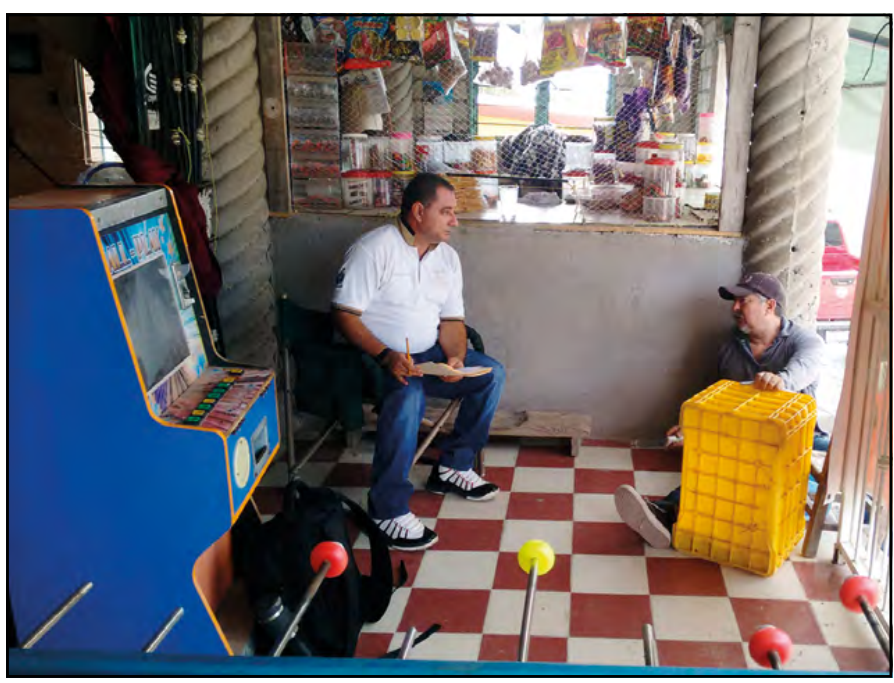

Figura 2. Entrevistas personales.

Imagen de Ramiro Ahumada Cervantes.

primero que viene a su mente cuando les mencionan este término. En el tercer aspecto, la pregunta es ¿Usted entiende lo que es el cambio climático? A los pobladores que contestaron positivamente esta interrogante, posteriormente se les cuestionó lo que entendían por $\mathrm{CC}$, con el propósito de medir su nivel de comprensión del tema (cuarta pregunta). Las causas que lo generan se tocan en la quinta pregunta y las acciones individuales que los habitantes encuentran necesarias para disminuir las emisiones de GEl se abordan en la sexta.

Para identificar la idea que tiene la población sobre los impactos del CC a nivel local, el cuestionamiento fue $\dot{\imath}$ Cómo repercute el cambio climático en su comunidad (medios de vida locales)? Finalmente, la última pregunta se desarrolló con el propósito de conocer la predisposición de la población para implementar pequeños cambios (acciones) en su forma de vida, enfocados a la mitigación y adaptación al CC. Es importante mencionar que antes de realizar esta pregunta se les explicaron brevemente los términos mitigación y adaptación.

Para el tamaño de muestra se consideró como universo de estudio los 3,592 pobladores que reporta el INEGI (2010) para las localidades de El Coloradito, El Caracol y El Huitussi. El número de encuestas a aplicar se determinó utilizando la fórmula 1, y se obtuvo un tamaño de muestra de 193 encuestas.

$$
\mathrm{n}=\frac{\mathrm{N} \times \mathrm{Z}^{2} \times \mathrm{p} \times \mathrm{q}}{\mathrm{d}^{2} \times(\mathrm{N}-1)+\mathrm{Z}^{2} \times \mathrm{p} \times \mathrm{q}}
$$




\section{IIVESTIGAGIÓn Y CUERCIA DE LA UNIVERSIDAD AUTÓnOMF DE RGUASCHLIETTES}

41

donde:

$n=$ Tamaño de muestra.

$N=$ Tamaño de la población (3592)

$Z=$ Nivel de confianza $(95 \%, \alpha=0.05)$

$p=$ Probabilidad de éxito, o proporción esperada (0.843)

$q=$ Probabilidad de fracaso $(q=1-p)$

$d=$ Precisión o error admitido (5\%)

La distribución de cuestionarios por localidad se definió tomando en cuenta la proporción de habitantes de cada una de ellas. Correspondieron 117 encuestas para El Huitussi (62 mujeres y 55 hombres), 56 para El Caracol (29 mujeres y 27 hombres) y 20 para El Coloradito ( 10 mujeres y 10 hombres). Es importante mencionar que se eligieron 193 viviendas al azar en dichas comunidades y la encuesta se aplicó a quien estuviera disponible para contestarla, siempre y cuando fuera mayor de 14 años.

\section{RESULTADOS}

\section{Percepción sobre cambios del clima en los últimos años}

De los encuestados $84 \%$ percibe cambios en el clima. Quienes más los perciben son los habitantes de El Caracol con 96\%, seguido de los de El Huitussi con $82 \%$ y los de El Coloradito con $65 \%$. Los hombres son quienes en mayor medida perciben cambios con $88 \%$ de la muestra, en comparación con las mujeres que en $81 \%$ dicen percibirlos; los resultados coinciden en las tres comunidades.

Los pobladores han observado cambios en cuatro aspectos relacionados con el clima: temperatura, precipitación, variación climática y vientos. Sin embargo, la temperatura es la variable más observada en las tres comunidades y representa $79 \%$ de la muestra total (figura 3).

\section{Conceptualización del cambio climático}

Solamente $10.4 \%$ de los encuestados ha recibido algún tipo de información o sensibilización referente al CC. En su mayoría corresponde a pláticas y talleres impartidos por la Comisión Nacional de Acuacultura y Pesca (CONAPESCA) o por la Secretaría de Desarrollo Social (SEDESOL). Dichos eventos son dirigidos a pobladores que tienen acceso a programas de apoyo pesquero $\bigcirc$ a programas sociales. La localidad que más sensibilización reporta es El Caracol con $22 \%$ y la que menos es El Coloradito (0\%). Se ha sensibilizado $14 \%$ de los hombres y $8 \%$ de las mujeres.

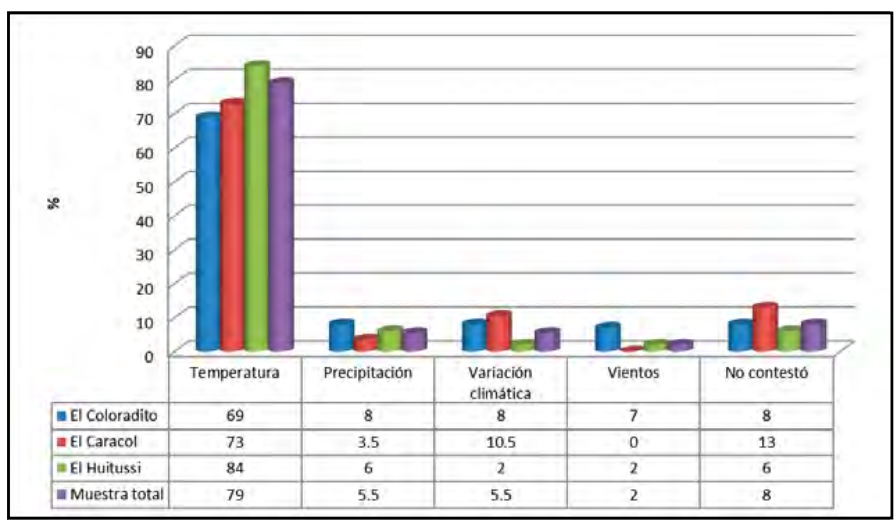

Figura 3. Aspectos del clima con cambios en los últimos años según la percepción de los pobladores (\%).

Elaboración propia.

Los pobladores asocian el CC con siete aspectos generales. Sin embargo, mucho calor y variación climática son los principales aspectos asociados al CC en la muestra total y coincide en las tres comunidades involucradas (figura 4).

Se consideró con un nivel de conocimiento aceptable a las personas encuestadas que han escuchadoeltérminocambioclimáticoylorelacionan correctamente con sus causas y consecuencias. En este sentido, $31.6 \%$ de la población mostró un nivel de conocimiento aceptable acerca del tema. Las comunidades El Coloradito y El Huitussi son las que mayor conocimiento tuvieron, ya que en ambas 35\% de los participantes arrojaron niveles aceptables. Con respecto a El Caracol, solamente $23 \%$ de la muestra exhibió un buen nivel de conocimiento. Asimismo, $35 \%$ de las mujeres y $28 \%$ de los hombres registraron niveles aceptables.

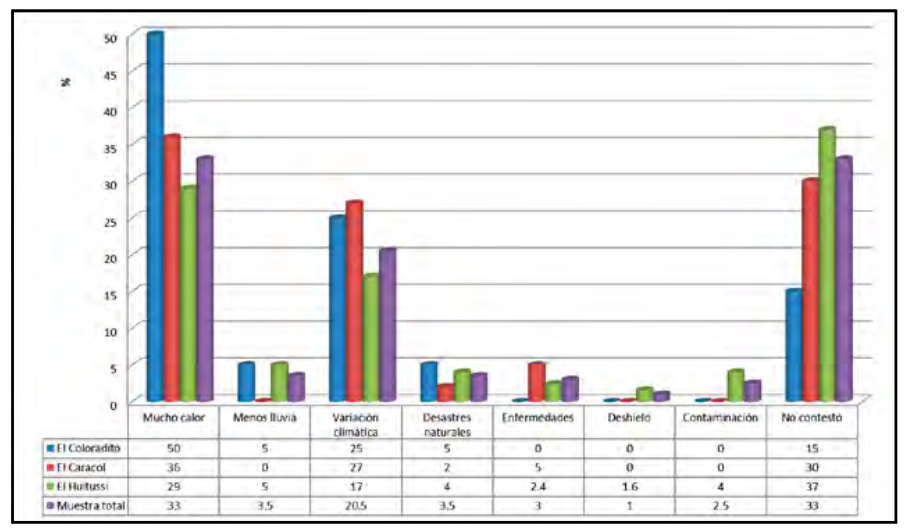

Figura 4. Aspectos con los que la población asocia al cambio climático (\%).

Elaboración propia. 
IIVESTIGACIÓn Y CIERCIA DE LA UחIVERSIDAD AUTÓNOTH DE RGUASCALIERTES
Las causas que generan el $\mathrm{CC}$ se agruparon en cinco aspectos: contaminación, incendios y deforestación, sobrepoblación, destrucción de la capa de ozono y causas naturales. Solamente $2.5 \%$ sitúa el origen del problema en causas naturales, mientras que la mayoría señaló causas antropogénicas. La mayoría sitúa el origen del CC en la contaminación (figura 5), esta apreciación coincide en las tres comunidades.

Las acciones individuales que los pobladores identifican para minimizar su contribución con emisiones de gases de efecto invernadero (GEI) se agruparon en tres aspectos: contaminar menos, reforestar y hacer conciencia. Aproximadamente la mitad coincide en que contaminar menos es la acción individual a implementar y lo enfocan en aspectos locales como el no quemar basura o no depositarla en lugares inadecuados para ello, así como el utilizar menos el automóvil. Los habitantes de las tres comunidades coinciden en sus respuestas para esta acción. En este sentido, $96 \%$ de los encuestados mencionaron estar dispuestos a implementar acciones encaminadas a la mitigación y adaptación al CC.

\section{Impacto del cambio climático en los medios de} vida locales

Los pobladores identificaron cuatro aspectos generales por medio de los cuales el CC tiene algún impacto negativo en sus medios de vida: enfermedades, menor producción, mal tiempo en el mar y mayor uso de aire acondicionado. Las respuestas más señaladas se muestran en la figura 6 .

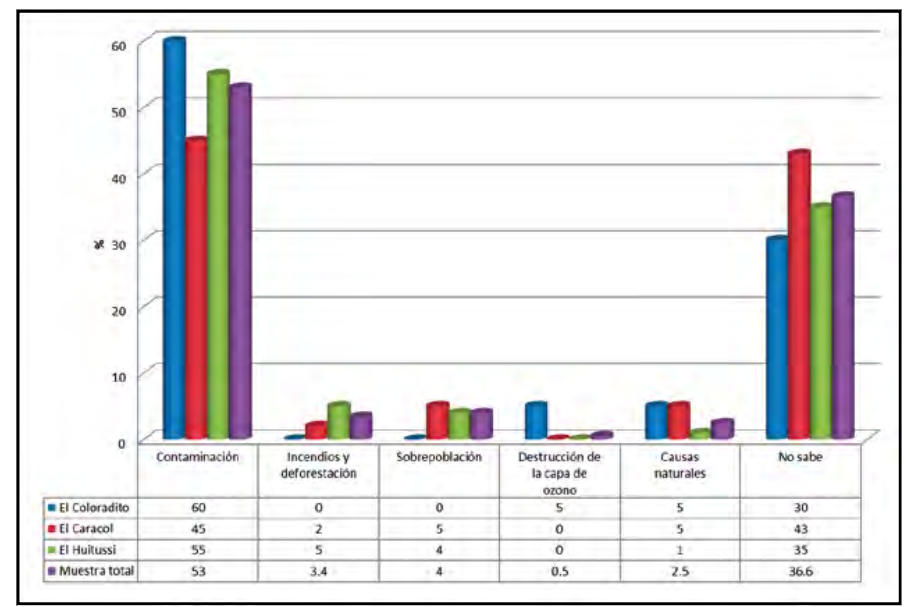

Figura 5. Causas del cambio climático según la percepción de los pobladores (\%).

Elaboración propia.

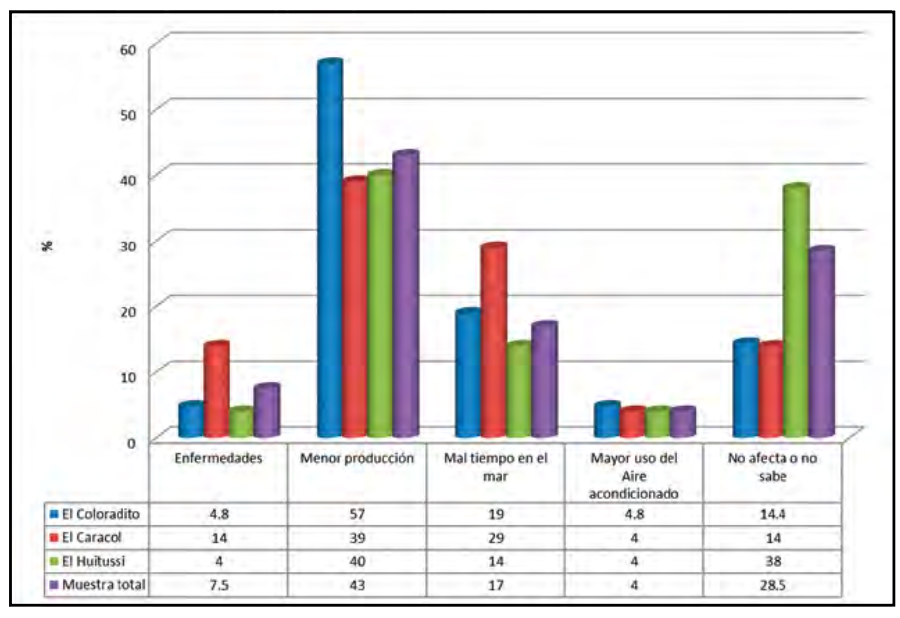

Figura 6. Impactos del cambio climático en los medios de vida según la percepción de los pobladores (\%).

Elaboración propia.

DISCUSIÓN

Aun cuando el conocimiento de los cambios pasados en el clima y las proyecciones a futuro han avanzado en las últimas décadas, este tipo de información solamente es conocida por una parte reducida de la población. Los pequeños cambios en las variables climáticas regularmente son percibidos por los pobladores locales, ya que en el desarrollo de sus actividades están expuestos a los elementos climáticos. En este sentido, 84\% percibieron cambios en los últimos años. Los resultados son similares a lo reportado por Oltra, Solá, Sala, Prades, \& Gamero (2009) mediante grupos de discusión integrados por personas no expertas en el tema; Soares \& MurilloLicea (2013) y Shameem et al. (2015) con muestras que incluyen comunidades costeras, y para estudios realizados en comunidades rurales que desarrollan actividades primarias, principalmente (Haq \& Ahmed, 2017; Hitayezu, Wale, \& Ortmann, 2017; Monirul Alam, Alam, \& Mushtaq, 2017; Panda, 2016).

Los hombres son quienes en mayor medida perciben estos cambios, lo cual es entendible, ya que al ser pescadores están más expuestos a los elementos y pueden percibir más el cambio en el clima. Estos resultados coinciden con los de Soares \& Murillo-Licea (2013).

La temperatura es la principal variable que observa cambios por los pobladores. Esto coincide con los informes científicos del IPCC (2014) en que la temperatura ha ido en aumento en las últimas décadas. Los resultados son similares a lo reportado 


\section{IIVESTIGAGIÓn Y CUERCIA DE LA UחIVERSIDAD AUTÓNOMA DE RGUASCALIERTES}

por Haq \& Ahmed (2017), Monirul Alam et al. (2017), Panda (2016) y Soares \& Murillo-Licea (2013).

Solamente $10.4 \%$ ha recibido alguna sensibilización relacionada con el CC. Este porcentaje se observa demasiado bajo, en relación con Soares \& Gutiérrez (2011), que reportan $52 \%$ de sensibilización para un municipio costero de Yucatán. Esto probablemente incide en el conocimiento que la población pueda tener sobre el tema. Básicamente, la sensibilización en la materia ha sido proporcionada por instituciones de gobierno y los hombres, al ser quienes mayormente reciben apoyos gubernamentales, son los más sensibilizados al momento. Esto coincide con los resultados de Soares \& Gutiérrez (2011) para un municipio de Yucatán.

Los pobladores asocian el CC con mucho calor, menos lluvia, variación climática, desastres naturales, enfermedades, deshielo y contaminación. Estos son aspectos recurrentemente mencionados en los diferentes estudios sobre percepción al CC (Haq \& Ahmed, 2017; Meira-Cartea \& Arto-Blanco, 2014; Monirul Alam et al., 2017; Oltra et al., 2009; Soares \& Gutiérrez, 2011). Mucho calor y variación climática son los principales aspectos que la población asocia con el CC, lo cual coincide con los hallazgos del IPCC (2014).

De la población encuestada, 65\% argumentó entender el término CC. Sin embargo, cuando se le cuestionó sobre ese conocimiento, solamente $31.6 \%$ mostró un nivel aceptable. Este es un porcentaje ligeramente arriba (26.7) de lo reportado por Nanlohy ef al. (2015) para un conjunto de comunidades costeras en la Bahía de Kotania. En este aspecto la mayoría de los estudios reportan porcentajes mayores a $50 \%$.

Es importante señalar que la idea principal que se tiene acerca del CC es el aumento en la temperatura, lo cual coincide con las observaciones de Soares \& Gutiérrez (2011). El bajo nivel de conocimiento del tema es coherente con la poca sensibilización recibida. Probablemente sus conocimientos se concretan a lo aportado por medios de comunicación. Al respecto, Vignola, Klinsky, Tam, \& McDaniels (2013) reportan que la mayoría de la población recibe información sobre el CC por la televisión, seguida por la radio y el diario.

Una parte de la muestra señaló el origen del CC en causas antropogénicas, lo cual coincide con Meira-Cartea \& Arto-Blanco (2014), Vignola et al. (2013) y Yu, Wang, Zhang, Wang, \& Wei (2013) y es coherente con los hallazgos del IPCC $(2013,2014)$. De acuerdo con los estudios de Oltra et al. (2009), el CC aparece vinculado principalmente a la contaminación, ya que es más fácil de entender para los individuos. En este sentido, la mayor causa generadora del CC es la polución y la principal acción individual a implementar es contaminar menos. A la contaminación generadora del CC la asocian principalmente con un problema local, a nivel micro, como la quema de basura, sin analizar la situación a nivel global con la gran diversidad de fuentes emisoras de GEl que existen en el planeta.

El CC repercute en los medios de vida locales (según la percepción de la población) provocando enfermedades ocasionadas por las condiciones inclementes del tiempo o por fauna nociva atraída por las nuevas condiciones establecidas por el CC; menor producción pesquera, agrícola o ganadera, dependiendo de la actividad desempeñada; mal tiempo en el mar, lo que dificulta desempeñar la actividad pesquera; mayor uso del aire acondicionado, ocasionado por el aumento de la temperatura. Las respuestas más señaladas por los pobladores fueron menor producción y mal tiempo en el mar. Los resultados son consistentes con los de Olmos Martínez et al. (2013) y con los de Sandoval et al. (2015). En el mismo sentido, la literatura menciona como aspectos críticos relacionados con el CC en comunidades costeras el aumento de la frecuencia e intensidad de los eventos extremos y cambios en la disponibilidad de recursos pesqueros (Bennett et al., 2016; Colburn et al., 2016; Nanlohy et al., 2015).

El desconocimiento sobre los principales aspectos relacionados con el CC se hace evidente al observar las figuras 4,5 y 6 . Se aprecia que un tercio de la población no contestó. En este sentido, no reconocen los aspectos relacionados al CC, no conocen las causas que los originan ni cómo impacta en su medio de vida. Sin embargo, se muestran receptivos para recibir información relacionada con el tema y para contribuir con acciones enfocadas en la mitigación y adaptación.

Los resultados obtenidos son interesantes y útiles como base para diseñar estrategias de sensibilización e involucramiento de la población enfocadas en la mitigación y adaptación al CC a nivel local. Lo anterior como complemento a los estudios de vulnerabilidad y adaptación que también se están desarrollando en la región de estudio. 
IIVESTIGACIÓn Y CIERCIA DE LA UNIVERSIDAD AUTÓNOMA

44 DE RGUASCALIERTES
CONCLUSIONES

De la población encuestada, $84 \%$ percibe cambios en el clima en los últimos años, principalmente en la temperatura. Los resultados coinciden en las tres comunidades y generalmente los hombres perciben más estos cambios.

Los pobladores asocian el CC con el calor y la variación climática, principalmente. Sin embargo, se nota un desconocimiento del problema, ya que más de $30 \%$ no respondió las preguntas y solamente $31.6 \%$ mostró un nivel de conocimiento aceptable acerca del CC. Esto puede estar asociado a que únicamente $10.4 \%$ ha recibido pláticas, talleres o clases referentes al CC.

Según la apreciación encontrada, la principal causa generadora del CC es la contaminación. Desde su punto de vista, este fenómeno afecta los medios de vida locales de dos maneras principalmente: 1) genera mal tiempo en el mar, lo que dificulta las pesquerías y 2) disminuye la producción pesquera, agrícola y ganadera. Para contrarrestar el problema, $96 \%$ está dispuesta a implementar medidas de mitigación y adaptación al CC. Por lo pronto, la principal acción que identifican y pueden implementar a corto plazo para disminuir el CC es contaminar menos.

Los resultados obtenidos son útiles como base para diseñar estrategias de sensibilización e involucramiento de la población enfocadas en la mitigación y adaptación al CC a nivel local.

\section{Agradecimientos}

Los autores desean agradecer a la Secretaría de Educación Pública (SEP), quien a través del Programa para el Desarrollo Profesional Docente para el tipo Superior (PRODEP) aportó el financiamiento del proyecto "Exploración de la vulnerabilidad y la percepción ante el cambio climático de la población rural del municipio de Guasave, Sinaloa" en el rubro de apoyo de fomento a la generación y aplicación innovadora del conocimiento.

\section{REFERENCIAS}

- $\quad$ Arbuckle, J. G., Morton, L. W., \& Hobbs, J. (2013). Farmer beliefs and concerns about climate change and attitudes toward adaptation and mitigation: Evidence from lowa. Climatic Change, 118(3-4), 551-563.

- Bennett, N. J., Blythe, J., Tyler, S., \& Ban, N. C. (2016). Communities and change in the anthropocene: Understanding social-ecological vulnerability and planning adaptations to multiple interacting exposures. Regional Environmental Change, 16(4), 907-926.

- Carlton, S. J., \& Jacobson, S. K. (2013). Climate change and coastal environmental risk perception in Florida. Journal of Environmental Management, 130, 32-39.

- Colburn, L. L., Jepson, M., Weng, C., Seara, T., Weiss, J., \& Hare, J. A. (2016). Indicators of climate change and social vulnerability in fishing dependent communities along the Eastern and Gulf Coasts of the United States. Marine Policy, 74, 323-333.

- Hagen, B., Middel, A., \& Pijawka, D. (2016). European climate change perceptions: Public support for mitigation and adaptation policies. Environmental Policy and Governance, 26(3), 170-183.

- Haq, S. A., \& Ahmed, K. J. (2017). Does the perception of climate change vary with the socio-demographic dimensions? A study on vulnerable populations in Bangladesh. Natural Hazards, 85(3), 1759-1785.
- Hitayezu, P., Wale, E., \& Ortmann, G. (2017). Assessing farmers' perceptions about climate change: A double-hurdle approach. Climate Risk Management, 17, 123-138. doi: 10.1016/j. crm.2017.07.001

- Instituto Nacional de Estadística y Geografía. (2009). Prontuario de información geográfica municipal de los Estados Unidos Mexicanos. Recuperado de http://www.inegi.gob.mx

- (2010). Censo General de Población y Vivienda 2010. México: Autor.

- Meira-Cartea, P. A. (2008). Comunicar el cambio climático. Escenario social y líneas de acción. Madrid: Ministerio de Medio Ambiente y Medio Rural y Marino-Organismo Autónomo de Parques Nacionales.

\& Arto-Blanco, M. (2014). Representaciones del cambio climático en estudiantes universitarios en España: Aportes para la educación y la comunicación. Educar em Revista, 3, 15-33.

- Monirul Alam, G. M., Alam, K., \& Mushtaq, S. (2017). Climate change perceptions and local adaptation strategies of hazard-prone rural households in Bangladesh. Climate Risk Management, 17, 52-63. doi: 10.1016/j.crm.2017.06.006 


\section{IIVESTIGACIÓn Y CIEREIA DE LA UחIVERSIDAD AUTÓNOMA DE RGUASCALIERTES}

- Nanlohy, H., Bambangb, A. N., Ambariyanto, \& Hutabarat, S. (2015). Coastal communities knowledge level on climate change as a consideration in Mangrove Ecosystems Management in the Kotania Bay, West Seram regency. Procedia Environmental Sciences, 23, 157-163.

- Olmos Martínez, E., González Ávila, M. E., \& Contreras Loera, M. R. (2013). Percepción de la población frente al cambio climático en áreas naturales protegidas de Baja California Sur, México. Polis. Revista Latinoamericana, 35, 1-17.

- Oltra, C., Solá, R., Sala, R., Prades, A., \& Gamero, N. (2009). Cambio climático: Percepciones y discursos públicos. Prisma Social, 2, 1-23.

- Panda, A. (2016). Exploring climate change perceptions, rainfall trends and perceived barriers to adaptation in a drought affected region in India. Natural Hazards, 84 (2), 777-796.

- Panel Intergubernamental del Cambio Climático. (2013). Summary for Policymakers. En T. F. Stocker, D. Qin, K. Plattner, M. Tignor, S. K. Allen, J. Boschung, A. Nauels, Y. Xia, V. Bex, \& P. M. Midgley (Eds.), Climate Change 2013: The Physical Science Basis. Contribution of Working Group to the Fifth Assessment Report of the Intergovernmental Panel on Climate Change (pp. 3-29). New York, NY: Cambridge University Press. doi: $10.1017 / \mathrm{CBO} 9781107415324.004$

(2014). Summary for Policymakers. En B. B. Field, V. R. Barros, D. J. Dokken, K. J. Mach, M. D. Mastrandrea, T. E. Bilir, M. Chatterjee, K. L. Ebi, Y. O. Estrada, R. C. Genova, B. Girma, E. S. Kissel, A. N. Levy, S. MacCracken, P. R. Mastrandrea, \& L. L. White (Eds.), Climate Change 2014: Impacts, Adaptation, and Vulnerability. Part A: Global and Sectoral Aspects. Working Group Il to the Fifth Assessment Report of the Intergovernmental on Climate Change (pp. 1-1131). New York, NY: Cambridge University Press.

- Retamal, M. R., Rojas, J., \& Parra, O. (2011). Percepción al cambio climático y a la gestión del agua: Aportes de las estrategias metodológicas cualitativas para su comprensión. Ambiente \& Sociedade Campinas, 14(1), 175-194.
- Sandoval, C., Soares, D., \& Munguía, M. T. (2015). Vulnerabilidad social y percepciones asociadas al cambio climático: Una aproximación desde la localidad de Ixil, Yucatán. Sociedad y Ambiente, 1 (5), 7-24.

- Secretaría de Medio Ambiente y Recursos Naturales6 de junio de Diario Oficial de la Federación, segunda sección, 1-29.

- Shameem, M. I., Momtaz, S., \& Kiem, A. S. (2015). Local perceptions of and adaptation to climate variability and change: The case of shrimp farming communities in the coastal region of Bangladesh. Climatic Change, 133(2), 253-266.

- Soares, D., \& García, A. (2014). Percepciones campesinas indígenas acerca del cambio climático en la cuenca de Jovel, Chiapas-México. Cuadernos de Antropología Social, 39, 63-89.

- Soares, D., \& Gutiérrez, I. (2011). Vulnerabilidad social, institucionalidad y percepciones sobre el cambio climático: Un acercamiento al municipio de San Felipe, Costa de Yucatán. Ciencia Ergo Sum, 18(3), 249-263.

- Soares, D., \& Murillo-Licea, D. (2013). Gestión de riesgo de desastres, género y cambio climático. Percepciones sociales en Yucatán, México. Cuadernos de Desarrollo Rural, 10(72), 181-199.

- Tuler, S., Agyeman, J., Da Silva, P. P., LoRusso, K. R., \& Kay, R. (2008). Assessing vulnerabilities: Integrating information about driving forces that affect risks and resilience in fishing communities. Human Ecology Review, 15(2), 171-184.

- Vignola, R., Klinsky, S., Tam, J., \& McDaniels, T. (2013). Public perception, knowledge and policy support for mitigation and adaption to Climate Change in Costa Rica: Comparisons with North American and European studies. Mitigation and Adaptation Strategies for Global Change, 18(3), 303-323.

- Yu, H., Wang, B., Zhang, Y. J., Wang, S., \& Wei, Y. M. (2013). Public perception of climate change in China: Results from the questionnaire survey. Natural Hazards, 69(1), 459-472. 